\title{
Lightning exposure of Carbon Fiber Composites in wind turbine blades
}

\author{
Søren Find Madsen, Lisa Carloni \\ Global-Lightning Protection Services A/S \\ Hovedgaden 451 K, 2640 Hedehusene \\ sfm@glps.dk
}

\begin{abstract}
Wind turbines are more and more often erected in remote areas of the world, in order to exploit better wind conditions. In these areas the cost of failures and repairs can be substantial. For this reason ensuring the lightning performance of the turbines and especially of the blades has become very important.

Modern blades are to a large extent manufactured using Carbon Fiber Composite (CFC) structural parts, due to the CFC's excellent mechanical tensile strength and stiffness, combined with a light weight. However, Carbon Fiber Composites also exhibit highly anisotropic electric conductivities, which require special attention in terms of lightning protection, primarily in what concerns electrical bonding.

The present paper presents the latest findings on how to include CFC materials in wind turbine blades into the lightning protection coordination, both in terms of engineering analysis using modern numerical tools, as well as with experimental validation in the lightning test facility.

The paper is part of the EU funded project SPARCARB which started January $1^{\text {st }} 2015$ and which aims at exploring the details of lightning interactions with CFC materials, damage mechanisms, optimization of electrical/thermal properties by adjusting the chemical composition of resin, fiber sizing, weaving techniques, manufacturing processes, etc.
\end{abstract}

\section{Introduction}

Carbon fiber composites are currently being used in wind turbine blades for structural components as spar caps, as illustrated in Figure 1, as the central load carrying structure in blades utilizing the central beam, for connections between metal conductors and CFC, and for heating. In these applications different fiber properties are exploited. In the spars or beams, Unidirectional (UD) fiber plies, such as those illustrated in Figure 2 and Figure 3, with fibers in the blade lengthwise direction are used to ensure the maximum tensional strength along the length of the blade. Bi-axial (Biax) or tri-axial (Triax) plies are used for controlling the torsional stiffness and because of their waving patterns they are also used to ensure a controlled current density distribution for electrical connections. Finally woven carbon fibers, such as those shown in Figure 4, are used for heating purposes, exploiting the resistive nature of the CFC.
The different conductivity properties of the CFC in these implementations can be exploited to optimize the blade design.

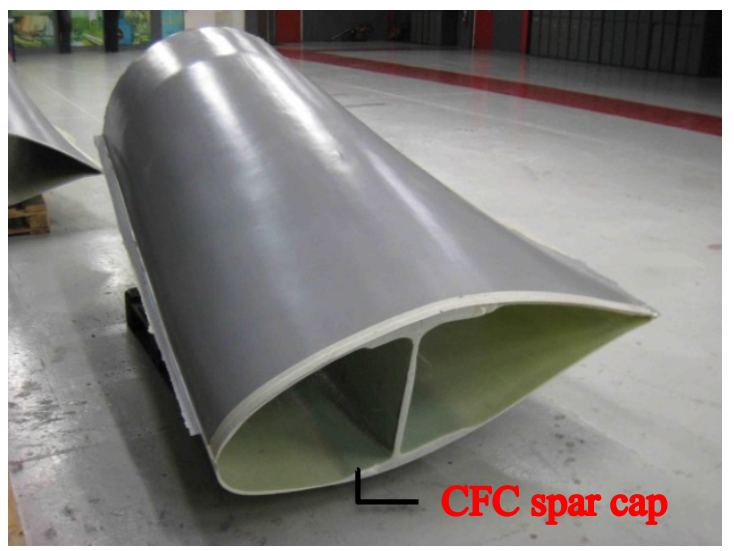

Figure 1 - Wind blade root section with carbon fiber composite spar (Source: ÉireComposites)

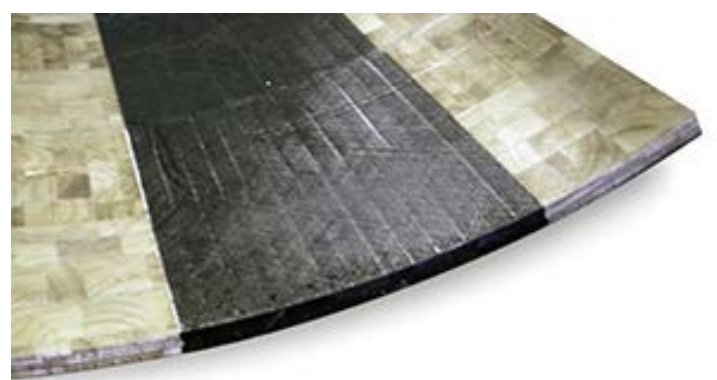

Figure 2 - UD carbon fiber spar cap (Source: Fiberline Composites)

For instance, the lightning current can be conducted along the length of the blade by UD fibers or spread over a surface by a CFC Biax.

This requires however a solid understanding of the conductivity properties of the CFC, of its thermal properties, of its failure mechanisms and of how these are affected by aging for instance. Since the use of CFC in the wind industry is relatively recent, compared with the automotive or aviation industry, the guidelines on how to define, detect, diagnose or test the failure modes still need to be established.

In wind turbine blades, a new set of industry-specific requirements arise: since the turbines are often located in remote locations, and some components are difficult or expensive to reach during service, the blades need to be able to operate with limited maintenance. For lightning protection, this implies coordinating air terminations, lightning current paths, shorts, surge protection devices and insulation, so that the blade can 
at least withstand the expected number of lightning strikes between services.

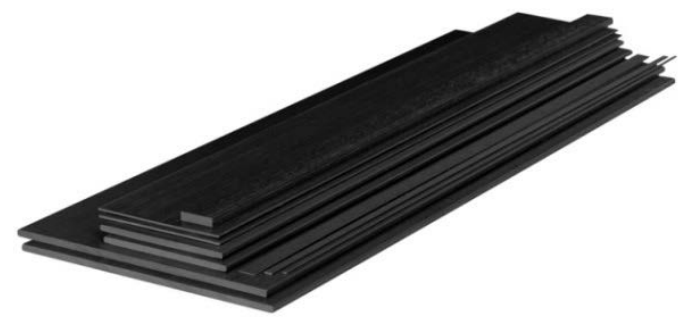

Figure 3 - Pultruded pre-cured UD carbon fiber (Source: Zoltek, Toray Group)

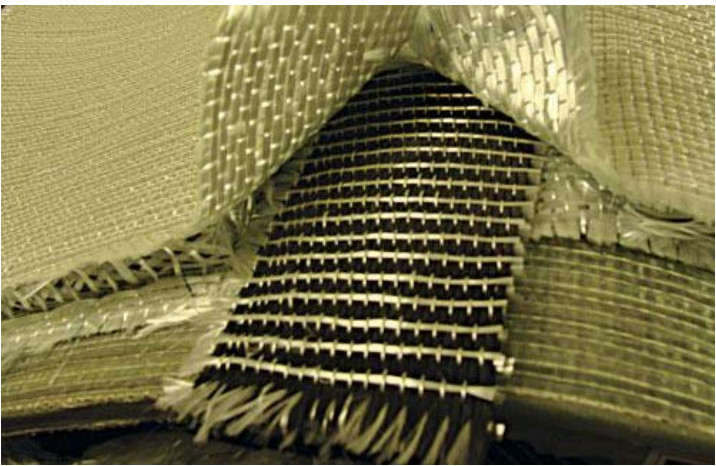

Figure 4 - Woven carbon fibers (Source: Composites World)

\section{Lightning exposure}

Lightning protection of wind turbine blades can be divided into two main concerns, lightning interception, and lightning current conduction. The first defines where the blade is struck, and hence fixes the position and design of the lightning air termination system.

The assessment of which areas of the wind turbine blades are most exposed to direct lightning strikes, and which amplitudes are most likely to be experienced by the different parts of the blade is based on numerical modeling and field surveys [1] [2]. The findings can be summarized by the 'Zoning concept' illustrated in Figure 5.

The blade is sectioned into different exposure areas. The first meter from the tip is the most exposed, with the highest probability of strikes and currents up to $200 \mathrm{kA}$, while the region between the root and $15 \mathrm{~m}$ from the tip is only exposed to currents below $10 \mathrm{kA}$.
One of the issues which needs to be considered when designing blade LPS, is the probability of having strikes of different amplitudes. This means that even if a strike of a certain type may cause very severe damage, it might be that the probability of having such a strike is insignificant, as shown by the probability density functions in the IEC 62305-1 [3]. For instance the probability of stroke amplitudes being smaller than 3kA or above $200 \mathrm{kA}$ is only app. $2 \%$.

Combining the probability distribution of having a strike to a given part of the blade [1] with the probability of having a lightning strike of a certain amplitude [3], it is possible to derive the exposure rates for the different parts of the blade [1].

\section{Lightning exposure and damage patterns in CFC blades}

Compared with the damage distribution experienced by Glass Fiber Reinforce Plastic (GFRP) blades [2], the damage distribution in CFC blades appears quite similar, indicating that the same lightning environment and methodologies can be used to assess the lightning attachment point distribution.

Damages experienced on CFC blades can occur for the same reasons as for GFRP blades, but may also be caused by direct attachment to the CFC structures, explained by inadequately placed air terminations (receptors) and poor insulation of CFC parts especially in the tip region (outermost $3 \mathrm{~m}$ of the blade).

Another cause of failure for CFC blades is the lack of, or inadequate equipotential bondings between the conductive paths, in particular between spars and the other conductive paths [2].

\section{Analysis of lightning current conduction effects in CFC applications}

As mentioned in the previous section, lightning protection of wind turbine blades can be divided into lightning interception, and lightning current conduction, i.e. the correct placement of air terminations (receptors) and the controlled conduction of current from the air terminations to the blade root.

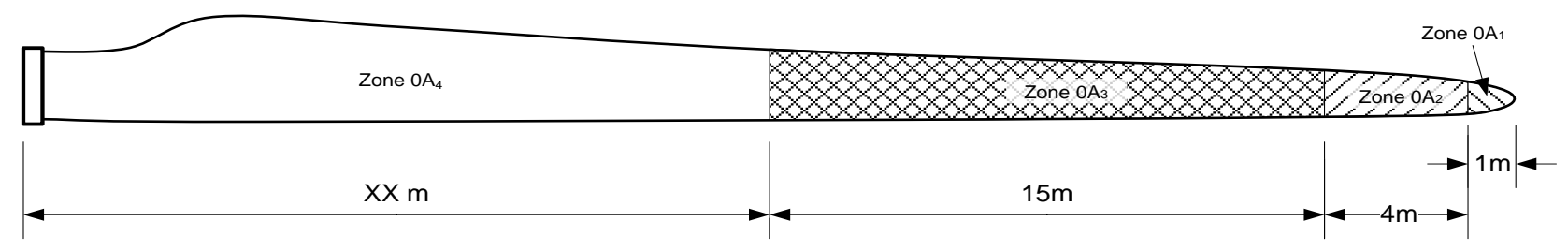

Zone $\mathrm{OA}_{1}$ : Tip end to $1 \mathrm{~m}$ inboard, $<200 \mathrm{kA}$

Zone OA2: $1 \mathrm{~m}$ inboard to $5 \mathrm{~m}$ inboard, $<100 \mathrm{kA}$

Zone $\mathrm{OA}_{3}: 5 \mathrm{~m}$ inboard to $20 \mathrm{~m}$ inboard, $<50 \mathrm{kA}$

Zone OA4: $20 \mathrm{~m}$ inboard to root end, $<10 \mathrm{kA}$

Figure 5 - Illustration of the 'Zoning concept'. The blade is sectioned into different exposure areas. The first meter from the tip is the most exposed, with currents up to $200 \mathrm{kA}$, while $15 \mathrm{~m}$ from the tip only currents below $10 \mathrm{kA}$ attach. 
The lightning exposure assessment does not define the exact position of the air terminations, but rather give indications about the current amplitudes to be expected for the air terminations in a given blade radius. The exposure rates are used as input for the numerical modeling of the current conduction from the attachment point to the root of the blade. This analysis, called 'lightning protection coordination', requires an accurate analysis of the inner components of the blade, of how the lightning current distributes among blade components, of the temperature rise it causes in the components, and of the voltages induced by the lightning current between the different conductive paths.

To determine which parts of the blade require additional insulation or electrical bonding, it is first of all necessary to pinpoint the risks, namely flashovers between components, or punctures through insulation, due to large electric fields between the components, arcs, sparking and burns due to high current densities and specific energies. When several CFC structures and metal down conductors are present together with cables for sensors and other electrical devices, the complexity of the electrical system is such that this demands numerical modeling.

The models are generally used to identify critical areas, to optimize the design, to determine test specifications, and to infer electrical and thermal properties from measurements.

\subsection{Mechanical and electrical properties of CFC} Before addressing the issue of lightning current conduction in blades, it is worthwhile to summarize the electrical and mechanical properties of CFC.

Carbon fiber composites consist of carbon fibers infused with resin, combining the excellent tensile strength of the fibers, illustrated in Figure 6, with the adhesive properties and resistance to degradation of the resin in Figure 7, yielding an overall density comparable with that of glass fiber, as shown by Figure 8 .

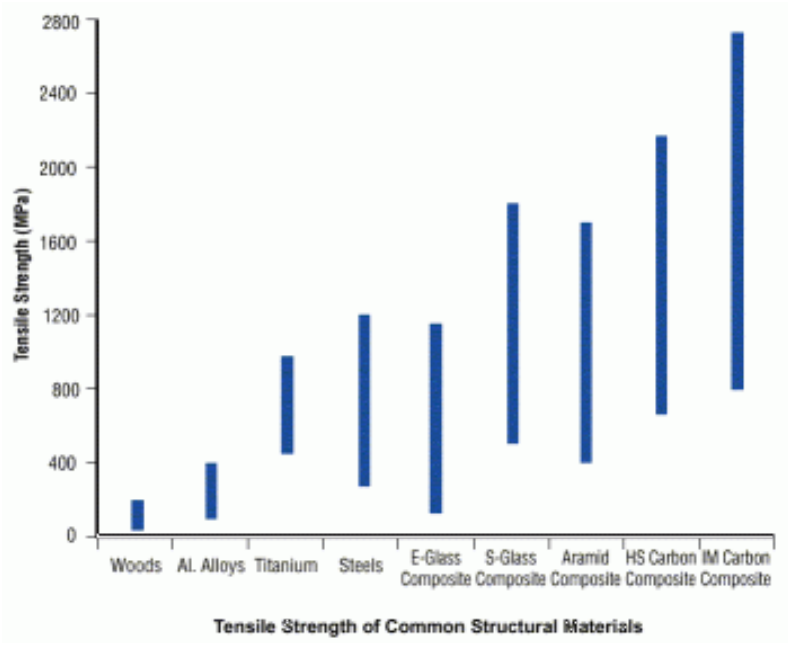

Figure 6 - Tensile strength of CF.

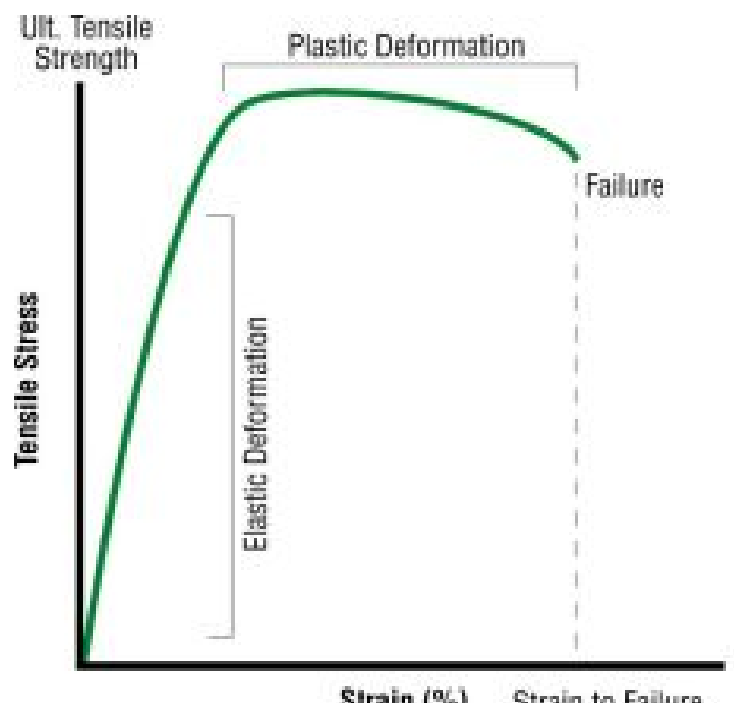

Figure 7 - Tensile strength for resin.

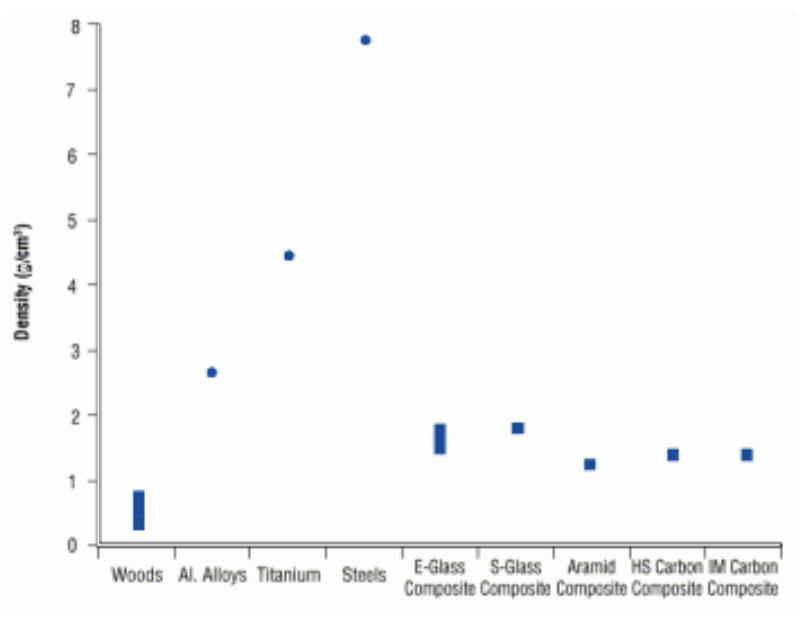

Densities of Commen Structural Materials

Figure 8 - Density for commercial structural materials.

As anticipated in the introduction, CF fibers are assembled in a variety of ways to better exploit these characteristics. Figure 9 shows an example of UD fibers, in which case the fibers are aligned.

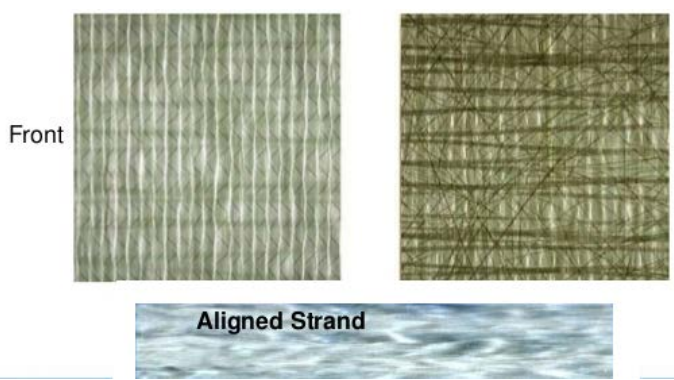

Back

Figure 9 - UD CF fibers (Source: Sandia 2014 Wind Turbine Blade Workshop- Miller \& Mandell).

Other examples are given by Biax CFC, where two sets of fibers are laid at an angle, as shown by Figure 10. 


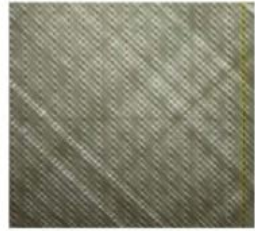

VU 90079

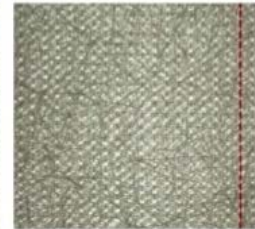

SX-1708

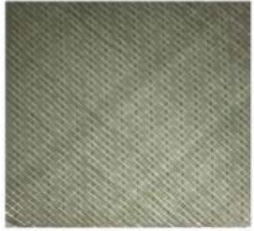

DB810-E05

Figure 10 - Biax CF fibers (Source: Sandia 2014 Wind Turbine Blade Workshop- Miller \& Mandell).

The electric and thermal properties are highly dependent on the direction of the fibers. As a rule of thumb, the conductivity across the fibers is $10^{3}$ to $10^{4}$ times worse than along the fibers. For a CFC assembled by Triax plies, the AC conductivity in plane with the fibres is roughly 1000 times worse than that of aluminum, but more or less constant with frequency due to the very limited skin effect experienced for CFC.

Similarly the thermal conductivity of CFC is rather anisotropic, and roughly 10 times higher along the fibers than across them (roughly $5 \mathrm{~W} \cdot \mathrm{m}^{-1} \cdot \mathrm{K}^{-1}$ against 0.5 $\left.\mathrm{W} \cdot \mathrm{m}^{-1} \cdot \mathrm{K}^{-1}\right)$.

The electric and thermal properties of the CFC are highly dependent on the manufacturing process. For example, Figure 2 and Figure 3 show two examples of UD fibers, one conventional, in which the spars are assembled by laying up sheets of UD fibers 'wet in wet' and one in which the CF is pre-cured. The electrical conductivity matrix for the two processes can differ by a factor 5. Individual measurements are therefore necessary for each material.

\subsection{Effects of lightning currents on CFC samples}

Lightning currents on CFC can cause mechanical breaking (excess of tension), delamination, erosion and burns. The causes are mostly due to the heat developed by the current entering the CFC, especially if a flashover or an arc occur, due to the charge depletion and - to a lesser extent - to the magnetic force between two conductors.

Given the intrinsically stochastic nature of arcs, flashovers and material breakdown, it is not possible to predict where or when a failure may occur. It is however possible to model a system to

1. to identify critical areas in the sample before tests are performed

2. to optimize design parameters such as CFC width, thickness, conductivity, interface resistance between CFC and conductors

3. to extract electrical and thermal properties from measurements

Modeling CFC samples allows a study of different parameters on a simplified system, rather than on the whole blade. Coupons are also more easily manageable

so they can be tested in the laboratory and results can be verified by comparing the expected and measured results. An example is illustrated in Figure 11 and Figure 12. Figure 11 shows the temperature rise in CFC Biax coupon due to the injection of a current pulse with $I_{\text {peak }}=200 \mathrm{kA}$, with a specific energy $\mathrm{AI}=3.37 \mathrm{MJ} / \Omega$ and Figure 12 shows the temperature rise that was recorded with a high speed thermography camera.

Other examples of coupon models involve CFC and metal interfaces, such as the one shown in Figure 15. When current is injected into the copper conductor, it passes through the interface, to the CFC, it propagates though the CFC and then back out through another similar interface and copper conductor. Given the anisotropy of the CFC conductivity matrix and the different materials involved, extracting the single impedances in highly non-trivial.

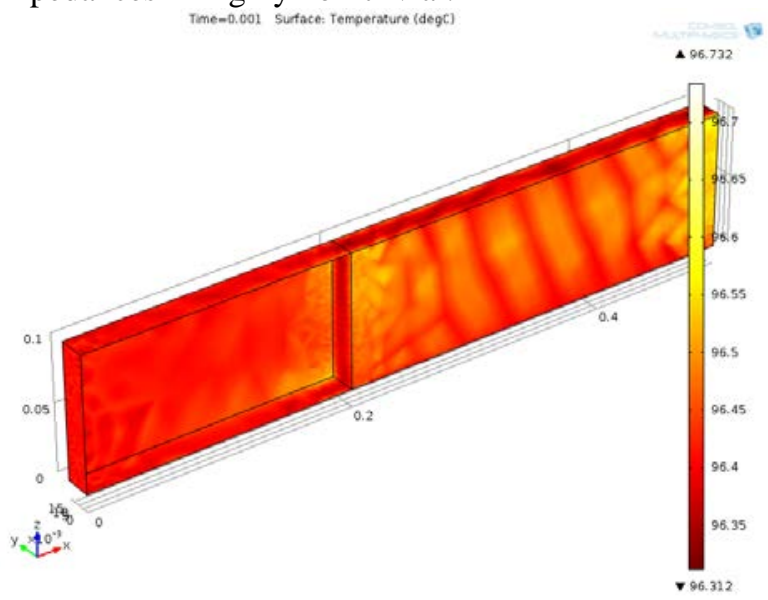

Figure 11 - Model of the temperature rise induced in a CFC coupon by lightning current. The results showed a temperature rise of $96^{\circ}$ after $1 \mathrm{~ms}$.

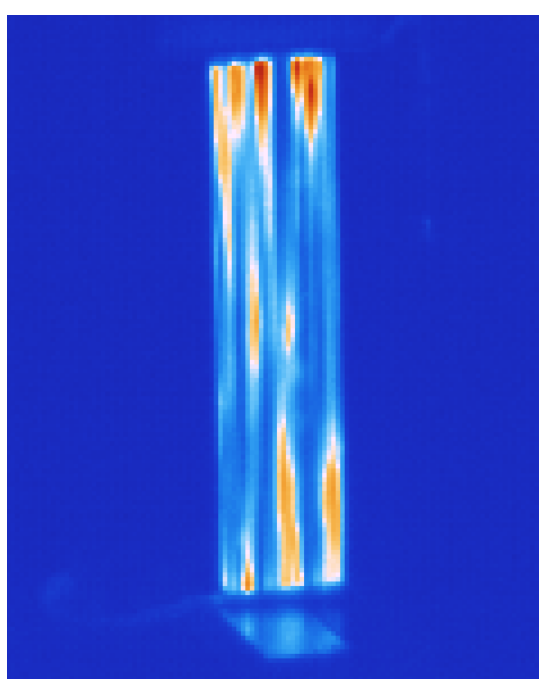

Figure 12 - IR image of the measured temperature rise in the corresponding test coupon. The results matched the expectations within a $10 \%$ error.

4.3. Full blade lightning protection coordination For full blades the main issue is the controlled conduction of the lightning current from the blade tip 
and the air terminations to the root end of the blade, avoiding flashovers, puncture, sparking and burns.

According to the international standard IEC 61400-24 concerning lightning protection of wind turbines [4], all conductive elements within the blades need to be electrically bonded. Bonding to CFC materials entails dealing with several challenges, since the material itself is highly anisotropic in these blade applications. Due to this anisotropy, which involves both electrical and thermal properties, current and heat are spread mainly along the length of the fibers, so that critical current density points and high temperatures may arise where the lightning current enters and exits the structure.

By analyzing the materials thoroughly considering weaving patterns, it is possible to identify the areas prone to sparking and arcing during lightning current conduction.

The first step in modelling, is to determine the exposure level, i.e. the current amplitude and probability of attachment to the blade, based upon the local ground flash density, the height of the turbine, the length and the shape of the blade, as described by [1]. Once the risk assessment has determined which current amplitudes attach to which part of the blade, IEC 61400-24 standard [4] first return stroke and subsequent stroke currents (10/350 us and 0.25/100 us) are injected into the blade model tip and side receptors, so one may study the effects of the partial currents on the components. This is typically done by combining a finite element model of the blade parts, with all its material properties, and then include these properties into a lumped circuit model.

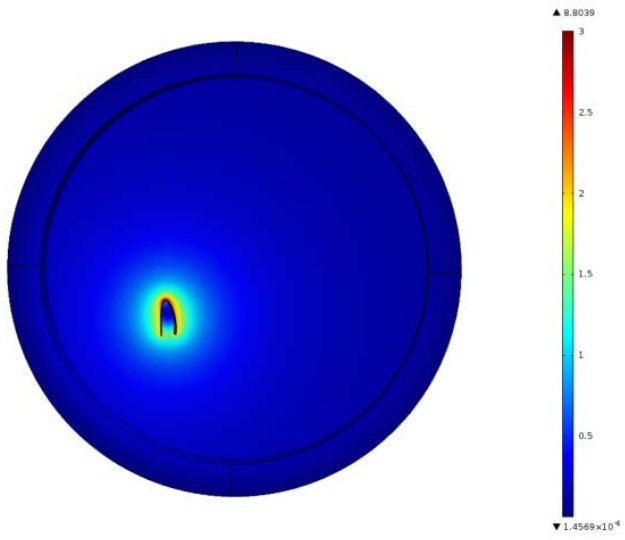

Figure 13 - Simulation of the magnetic field induced by the current flow in the heating mat in the surrounding air.

For the design of the CFC interfaces with the down conducting system, it is important to understand how the current and energy are shared between the different conductive paths. This kind of analysis allows to identify the critical design parameters, such as the peak current the solution must be able to withstand, and to compare different possible lightning protection solutions.

\section{Design}

Once the modeling of the samples has been conducted, the iterative process of designing solutions can start. The materials can be assembled into easily manageable coupons, which can be tested in the lab. For CFC this means assembling individual plies with the selected weaving pattern, and implementing the electric connections necessary for high current testing. The electric connections between CFC and metal down conductors, represent a critical issue. For small samples ( 1m long) it is possible to copper plate the surface, as shown in Figure 14, or to use conductive paints to obtain a low resistance interface, but for larger samples ( $\sim 50 \mathrm{~m}$ long) this is not feasible.
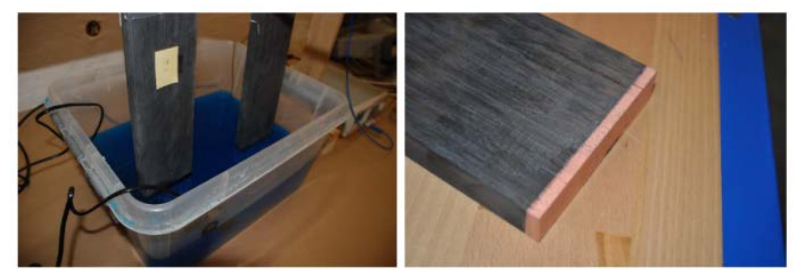

Figure 14 - Copper plating of a CFC sample. This requires connecting the two ends of a sample to electric leads, one of which is immerged in a solution together with a $\mathrm{Cu}$ bar. The current driven through the sample causes the $\mathrm{Cu}$ to deposit on the sample surface, thus creating a mechanical and electric connection $\mathrm{Cu}-\mathrm{CFC}$.

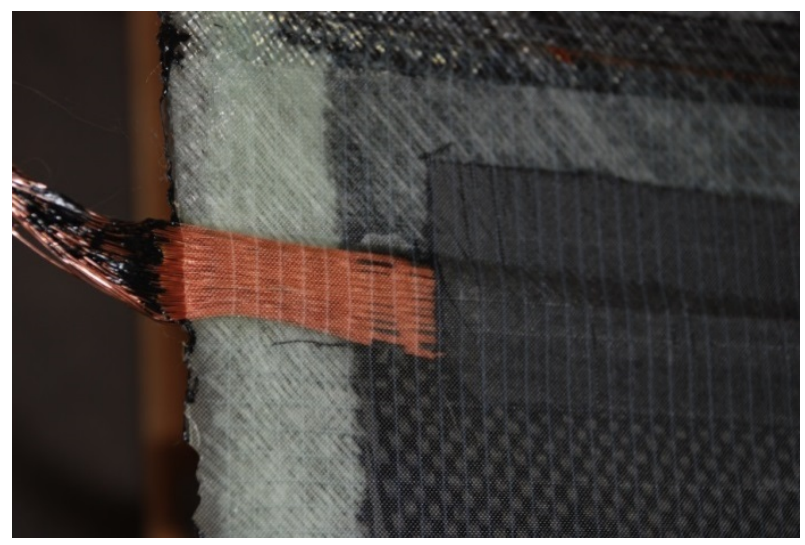

Figure 15 - A solution of the interface CFC to metal conductor for a large surface, including a combination of CFC Biax and woven metal conductor.

The main driver of the design phase is the optimization of the lightning protection solution. This may simply be the implementation of a rivet, or of a copper mesh interface, but it may also involve the development of resin systems for CFC with improved performances when exposed to lightning strikes.

\section{Laboratory testing}

The design process ends with a prototype (a coupon, a subsystem or a full blade) which is then verified in the lightning test laboratory. 
The test specifications are determined based on the exposure rates given by the simulations described in the previous section - an example of a typical high current test is shown in Figure 16. The expected lightning current pulse is injected into one end of the CFC sample and exits at the other end. The high current causes sparking along the length of the fibers and burns and delamination of the glass fiber outer layers (in white).

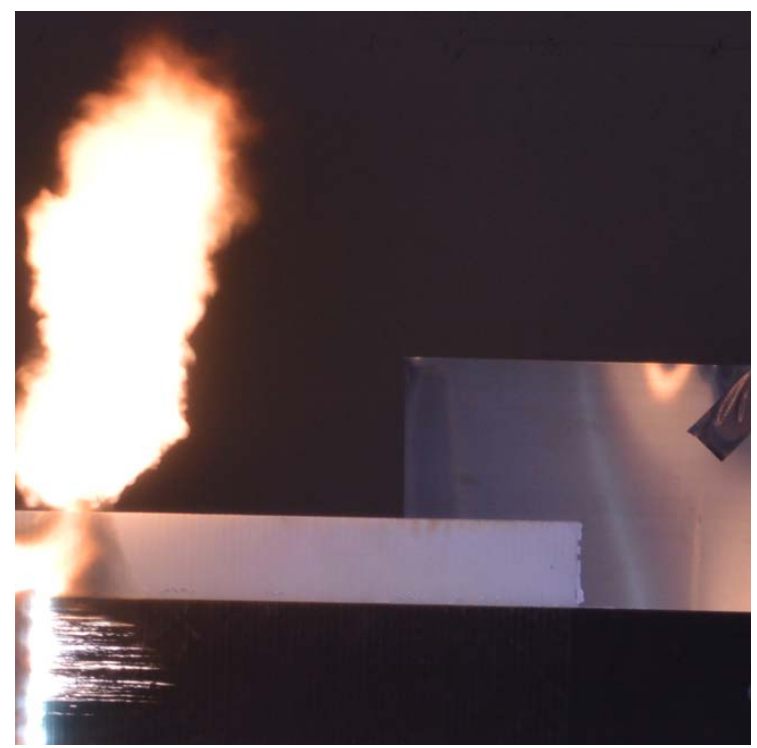

Figure 16 - High current test on a carbon fiber sample.

Following the exposure of the test coupon to the lightning environment, the manufacturer of the subsystem or the complete blade must document whether the severity of the impact compromised the structural integrity of the material. This analysis is currently done by visual inspection and resistance measurements, but the SPARCARB project will indeed cover these aspects in more detail.

\section{Performance evaluation}

As discussed in Section 6, there are no established practices for how to define, detect and diagnose the failure of a carbon fiber solution in the context of wind turbine blades. For instance, there is no established practice to quantify the damages due to lightning strikes on the carbon fibers. As yet, the very definition of the critical parameters that determine breakdown of the fibers is not established. It is customary to test the samples with the number of lightning impacts expected in a 20 year lifetime, based upon the ground flash density, the distribution of strikes with a given current amplitude, the current distribution in the single component assessed by the simulation, etc. It is however still not known whether the breakdown mechanism is a 'threshold effect' or a 'cumulative effect' (aging), i.e. whether it is the peak current, specific energy or charge in a single stroke that determine the breakdown or rather effect of the cumulated energy and charge.
One of the main scopes of the SPARCARB project is precisely the definition of the methodology and the identification of the parameters which determine a failure.

\section{Conclusion}

The present paper addresses the current issues of implementing lightning protection and CFC structural components in wind turbine blade manufacturing. The paper is part of a SPARCARB EU funded project within the Horizon 2020 framework, which will investigate the issues described in full detail during the years 20152018.

\section{References}

[1] A. G. B. S.F.Madsen, »'Lightning protection Zoning and Risk exposure assessment of wind turbines', « i 2015 EWEA Offshore, 2015.

[2] A. Garolera, 'Lightning Protection of flap system for wind turbine blades', $\mathrm{PhD}$ thesis, Technical University of Denmark, 2014.

[3] »IEC 62305-1 Ed. 2.0, 'Protection against lightning - Part 1: General Principles', « International Electrotechnical Commission, 2011.

[4] »IEC 61400-24 ed1.0 'Wind turbines - Part 24: Lightning protection, « International Electrotechnical Commission, ICS 27.180 ISBN 978-2-88910-969-2. 